\title{
Linear dichroism of DNA: characterization of the orientation distribution function caused by hydrodynamic shear
}

\author{
John C. Sutherland a, b, c
}

Keywords: DNA ligands, Couette cell, polarized absorption, scalene ellipsoid, photoelastic modulator, equivalent orthogonal orientation, dichroic increment ratio

\begin{abstract}
Linear dichroism provides information on the orientation of chromophores part of, or bound to, an orientable molecule such as DNA. For molecular alignment induced by hydrodynamic shear, the principal axes orthogonal to the direction of alignment are not equivalent. Thus, the magnitude of the flow-induced change in absorption for light polarized parallel to the direction of flow can be more than a factor of two greater than the corresponding change for light polarized perpendicular to both that direction and the shear axis. The ratio of the two flow-induced changes in absorption, the dichroic increment ratio, is characterized using the orthogonal orientation model, which assumes that each absorbing unit is aligned parallel to one of the principal axes of the apparatus. The absorption of the alienable molecules is characterized by components parallel and perpendicular to the orientable axis of the molecule. The dichroic increment ratio indicates that for the alignment of DNA in rectangular flow cells, average alignment is not uniaxial, but for higher shear, as produced in a Couette cell, it can be. The results from the simple model are identical to tensor models for typical experimental configurations. Approaches for measuring the dichroic increment ratio with modern dichrometers are discussed.
\end{abstract}

\section{Introduction}

Linear dichroism (LD) induced by laminar hydrodynamic flow gradients provides information on a variety of biological materials [1]. It has been used to study DNA [2-5] and ligands bound to DNA, including medicinal compounds [6-10] and carcinogens $[11,12]$. The early studies required large sample volumes and laborintensive experimental protocols. The recent increased interest in hydrodynamic shear induced LD for the study of biological systems has resulted, in part, from the commercial availability of Couette flow cells, consisting of coaxial cylinders one of which rotates, as shown in Figure $1[1,5]$. These cells require only small volumes of sample, which fills the space between the inner and outer cylinders. Another factor is the realization that a single spectrometer can be used for multiple types of measurements, including LD, circular dichroism (CD), magnetic CD (MCD), several types of fluorescence experiments $[13,14]$, plus the simultaneous measurements of absorption 
$[15,16]$. While originally laboratory built, such emission and polarization spectrometers have become commercially available, thus providing access to a wider range of users and reducing the incremental cost of obtaining the equipment needed to measure LD.

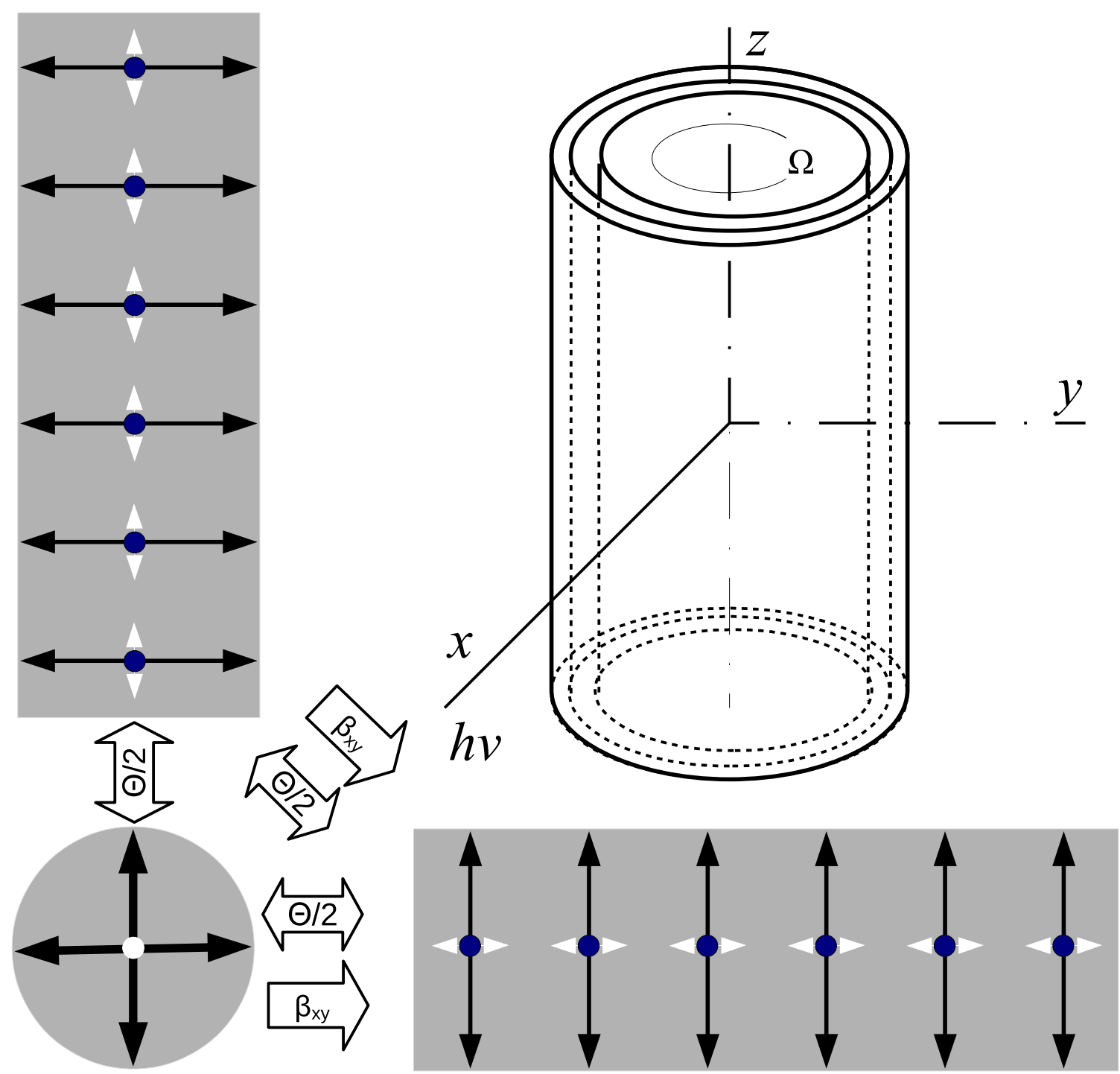

Figure 1. A perspective view of a Couette cell and schematic diagram of DNA molecules, shown in gray, aligned parallel to the $x, y$, and $z$ axes and viewed from a point on the $x$ axis. Molecules aligned parallel to the $x$ axis are represented by the circle. Molecules aligned parallel to the $y$ and $z$ axes are represented by the rectangle whose long axis is parallel to the corresponding axis in the perspective view. The projections of the dipole moments for optical transitions of the bases polarized parallel to the direction of the helix are shown in white, and the stronger components polarized perpendicular to the helix axis are in black. The rates at which the alignment probabilities are assumed to change are shown as labeled arrows. The direction of rotation of the core of the Couette cell is consistent with the shear gradient shown in Figure 2. 
This report demonstrates that some of the earliest studies of the LD of flow-oriented DNA inadvertently provided information on the spatial distribution of the partially aligned chromophores that is not immediately available from the latest generation of dichrometers, and explores ways in which such data might be obtained. The analysis is based on the equivalent orthogonal orientation model that corresponds to a subset of the parameters used to provide a general characterization of the distribution of chromophores in a flow-oriented population - only the diagonal matrix elements. They have the properties of probabilities, and are sufficient to characterize the data obtained in a typical LD experiment. Specifically, they can determine if flow-induced orientations in a particular experiment should be represented by a scalene ellipsoid (three unequal principal axes) or a prolate ellipsoid with rotational symmetry around the axis of orientation, i.e. if the orientation is uniaxial.

\subsection{A scalene distribution induced by hydrodynamic shear}

Understanding of the distribution of long molecules under the influence of a flow velocity gradient is facilitated by consideration of a rigid rod of length $2 r$, in a flow field, as shown in Figure 2. This description focuses on the shear pattern produced in a Couette cell, although some of the data discussed below were recorded with single-pass flow cells with rectangular cross-sections, which have a different distribution of shear. For both systems, the rod makes angles $\xi, \psi$, and $\zeta$ with respect to the $x^{-}, y^{-}$, and $z$-axes. The origin of the coordinate system in this figure is located in the center of the rod, only half of which is shown. The rod is in a viscous liquid flowing parallel to the $y$ axis with a uniform velocity gradient along the $x$ direction. The parallel arrows shown in the $x-y$ plane and parallel to the $x$ axis represent the difference in velocity of the fluid relative to the center of the molecule. These arrows represent a vector field that fills the entire sample space, with all of the relative velocity vectors having a magnitude that for a Couette cell increases linearly in proportion to the distance along the $x$ axis from the origin. For negative values of $x$, the directions of these vectors are reversed. Because the flow velocity increases linearly, the shear is constant along the $x$ axis. At each point along the rod, the relative flow shear exerts a torque on the rod proportional to the vector produce of the radius vector from the origin to the point on the rod and the differential flow vector at that location in the fluid. Thus for each point along the rod, both the magnitude of the shear and the lever arm through which it operates are proportional to $\operatorname{Cos}[\xi]$, so the torque is proportional to $\operatorname{Cos}^{2}[\xi]$. The resulting torque vector is perpendicular to the $r-y$ plane and the torque twists the rod so as to decrease the magnitude of the angle $\psi$, thus moving the rod in the direction of alignment with the direction of flow. The torque is a maximum for rods aligned parallel to the $x$ axis, decreasing monotonically for orientations closer to the $y-z$ plane. 


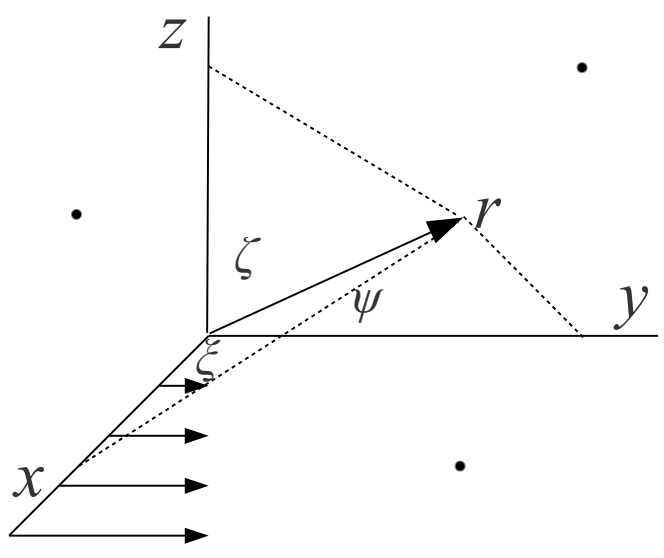

Figure 2. A uniform rigid rod shown in a gradient laminar-flow field. The origin of the coordinate system shown in the figure is located at the center of mass of the rod, only half of which is shown. The horizontal arrows indicate the hydrodynamic shear shown relative to the motion of the center of mass of the rod. If the inner cylinder of the Couette cell is rotating clockwise when viewed looking down the $z$ axis, the molecules are moving in the negative $y$ direction, creating a uniform velocity gradient proportional to $x$. The same relative shear would be generated if the inner cylinder is stationary and the outer cylinder rotates counterclockwise, but in that case, the molecules will be traveling in the positive $y$ direction. A light beam is traveling in the positive $x$ direction. The three angles are used to project the tip of the rod onto the corresponding axis, as indicated by the dotted lines. The three dots designate the projection of the tip of the arrow onto the $x-y, y-z$, and $z-x$ planes.

If the probability per unit solid angle, $\rho$, for finding a rod aligned in a given direction can be represented by an ellipsoid, it would be expressed as shown in (1), where the magnitudes of the probability densities along the semi-axes are $\rho_{\mathrm{y}}>\rho_{\mathrm{z}} \geqq \rho_{\mathrm{x}}$. The equality holds for a unidirectional alignment mechanism, such as induced by an electric or magnetic field, for which the orientation probability density is a prolate ellipsoid.

$$
\rho[\xi, \psi, \zeta]=\left(\frac{\operatorname{Cos}^{2}[\xi]}{\rho_{x}^{2}}+\frac{\operatorname{Cos}^{2}[\psi]}{\rho_{y}^{2}}+\frac{\operatorname{Cos}^{2}[\zeta]}{\rho_{z}^{2}}\right)^{-\frac{1}{2}}
$$

The analysis for rigid rods, presented above, would lead to a scalene ellipsoid, that is, an ellipsoid with three unequal principal axes. The values of these axial probability densities are constrained because the integral of $\rho$ over all solid angles must equal unity. For an isotropic system, all three must be equal to $\rho_{0}$. Since $4 \pi\left(\rho_{0}\right)^{2}=1$, it follows that $\rho_{0}=1 / 2 \sqrt{\pi} \approx 0.28$. The fact that laminar flow sheer induced orientation is not symmetric with respect to the $x$ and $z$ axes was known before the first use of shear-induced linear dichroism studies of DNA and other biomolecular systems, but frequently has been ignored (Section 3.2 of [1]).

DNA molecules cannot be treated as rigid rods because they are somewhat flexible. Therefore, part of the orientation process involves changes in shape as well as changes in orientation. The same is true of liposomes and some proteins. However, (1) may still be a reasonable description of the spatial distribution of the individual DNA segments, because the distribution function must morph continuously into a sphere in the absence of orienting forces. This report demonstrates how the dichroic 
increment ratio can be used to provide information on the distribution of flow-oriented molecules such as DNA and discusses differences in the information provided by some of the early experiments, cited above, that employed single pass flow cells, compared to the current generation of Couette cell equipped dichrometers. It also describes approaches for obtaining the information needed to calculate the dichroic increment ratio using modern dichrometers.

\section{Methods and conventions}

Digital values of data points in published charts were obtained using the Draw module of LibreOffice 5.2 (The Document Foundation, Berlin Germany). In addition to standard mathematical conventions, square brackets are used exclusively to enclose function arguments, braces indicate lists or collections, and parentheses are reserved for grouping terms. Multiple mathematical expressions are connected by right arrows, which can be read as "which implies." For expressions containing multiple steps, the arrows are numbered to facilitate referencing in the text, with an expression being identified by the number of the arrow preceding it.

\section{Theory}

DNA in solution is somewhat flexible, so the orientation of the entire molecule is never as well defined as it is for rigid rods. However, each short segment of the molecule can be considered a link in a chain, and the orientations of all such segments in all of the molecules defines the distribution of orientations of the ensemble. The orientation of a particular link is dependent on the links near it, but in the absence of an orienting stress, the steady-state probability of finding links oriented in a particular direction must on average be the same for all directions. Therefore, the optical properties of the ensemble are isotropic.

\subsection{Coordinate systems}

Analysis of LD requires definition of two coordinate systems. One is determined by the apparatus used in the measurement of LD, and is often referred to as the laboratory coordinate system. The other coordinate system is defined relative to the structure of the molecules or other structures that can be aligned. Previous reports and reviews have used a variety of terms and symbols to designate the axes in the laboratory coordinate system. Because there is no consensus, this report uses the nomenclature shown in Figure 1 and Figure 2, which was used previously to describe dichrometers [17]. Fluid flow at the points where the optical beam passes through the sample is parallel to the $y$ axis; the flow gradient and the propagation of light are parallel to the $x$ axis; the axis of rotation of a Couette cell, if present, is parallel to the $z$ axis, as is the force of gravity. The axis labels are similar to those used by Wada [18] and differ from those used Nordén et al. [1] in that the roles of the $y$ and $z$ axes are interchanged. The three angles that define the orientation of the rod are those used by Hofrichter and Eaton [19]. For the experiments performed with rectangular cross-section flow cells, the flow was usually in the positive $z$ 
direction - to prevent trapping of bubbles - but this report focuses on the Couette geometry, because these cells are employed in most recent experiments.

For the coordinate system of the orientable molecule, the symbol $\|$ is used to designate the axis parallel to the direction of orientation and the symbol $\perp$ to designate any axis perpendicular to the $\|$ axis. Thus, the standard presumptions of average cylindrical symmetry of short segments and lack of directionality along the orientable axis are inherent in the molecular coordinate system. While not significant for the present report, note that the lack of directionality means that care is required in constructing a complete probability distribution to avoid counting each molecule twice.

\subsection{Absorption}

The decadic absorption of an isotropic sample at a particular wavelength is given by $A=\log \left[I_{0} / I\right]$, where $I$ is the intensity of the transmitted beam when the sample is present and $I_{0}$ is the intensity of the transmitted beam of an appropriate blank. The exponential form of this expression is shown in (2). Certain derivations are simplified, however, if the Eulerian absorption, $a=\ln \left[I_{0} / I\right]$, is employed. As with the decadic absorption, the Eulerian absorption can be resolved at the product of three terms: $\sigma$, the molecular cross-section for absorption, which is an intrinsic parameter dependent on the absorbing species and is wavelength-dependent; $n$, the number of absorbers per unit volume; and $d$, the distance the light beam travels through the sample. All three depend on some power of distance $(2,-3$, and 1 , respectively), and it is assumed here that all are expressed using the same unit of distance. At the end of a derivation, it is easy to return to the decadic absorption (since $a=A \ln [10]$ ) and the usual product of terms, $\epsilon c d$, into which $A$ is resolved. For polymers, such as DNA and proteins, concentrations are usually expressed in terms of monomeric subunits, i.e., bases or amino acid residues, rather than the number of polymeric molecules.

$$
I=I_{0} 10^{-A} \stackrel{1}{\longrightarrow} I_{0} e^{-a} \stackrel{2}{\longrightarrow} I_{0} e^{-\sigma n d}
$$

In an LD experiment, the difference in absorption between light polarized parallel and perpendicular to the direction of preferred orientation is measured. For the coordinate system described above, the linear dichroism is given by the expression in (3), where $A_{\mathrm{y}}$ and $A_{\mathrm{z}}$ are absorption measured with light propagating parallel to the $x$ axis and polarized parallel to the $y$ and $z$ axes, respectively. The corresponding expression in terms of molecular absorption cross-sections are also shown. The reduced $\mathrm{LD}$ is the ratio of the $\mathrm{LD}$ divided by the isotropic absorption.

$$
\Delta A_{\mathrm{LD}} \equiv A_{\mathrm{y}}-A_{\mathrm{z}}=\Delta A_{\mathrm{yz}} \quad \Delta \sigma_{\mathrm{LD}} \equiv \sigma_{\mathrm{y}}-\sigma_{\mathrm{z}}=\Delta \sigma_{\mathrm{yz}}
$$

For characterization of the scalene ellipsoid, it is useful to define the dichroic increment ratio, $R_{\mathrm{l}}$, as shown in (4), where $A$ is the absorbance of the isotropic sample, and $\sigma$ is the corresponding isotropic cross-section. This ratio is dimensionless. The increment designation, which was introduced by Gray and Rubenstein $[3,20]$, is necessary because the term dichroic ratio has been used in several different ways by various authors. Both the linear dichroism and the 
dichroic increment ratio can also be expressed in terms of the corresponding Eulerian absorptions $(a)$ or molecular extinction coefficients $(\varepsilon)$.

$$
R_{\mathrm{I}} \equiv \frac{A_{y}-A}{A_{z}-A} \longrightarrow \frac{\sigma_{y}-\sigma}{\sigma_{z}-\sigma}
$$

Linear dichroism can provide useful information on the orientation of transition dipoles relative to the alignment axis. For the analysis of the distribution of alienable molecules subject to flow shear, a simple model is useful. The relationship of the parameters introduced in this model is connected to the expressions used in comprehensive theories of oriented systems later in this report.

\subsection{The spectroscopically equivalent orthogonal alignment model}

Suppose that each molecule or molecular segment in a sample, which on average is cylindrically symmetric, has a preferred axis that tends to be aligned by a hydrodynamic gradient or other mechanism. The average absorption cross-section for light polarized parallel to the alignment axis of the molecule is represented by $\sigma_{\|}$, while the coordinate system cross-section for light polarized perpendicular to the alignment axis is represented by $\sigma_{\perp}$. In the equivalent orthogonal orientation model all of the absorbing segments of the macromolecules in the sample are presumed aligned exactly parallel to one of the three principal axes of the laboratory system, as shown schematically in Figure 1. The alignment distribution of the system is defined by any two of the probabilities of finding a molecule or molecular segment oriented parallel to one of the three axes, which are represented by $p_{x}, p_{y}$, and $p_{z}$, respectively. For electronic transitions, the absorption cross-sections for light traveling parallel to one of these axes and with the polarization of the light parallel to one of the other principal axis can be expressed in terms of these parameters, and written by inspection of the oriented molecules shown in Figure 1. An alternate approach is to follow the rule that if the orientation of the molecules is parallel to the polarization of the light beam, i.e., the cross-section index and the probability index agree, the latter is multiplied by $\sigma_{\|}$. If they are perpendicular the probability is multiplied by

$\sigma_{\perp}$. Either approach leads to the results shown in (5). Although the primary interest in this report is on the arrangement in which light is traveling parallel to the direction of the shear gradient, which is the normal configuration of an LD experiments, the polarized absorption cross-sections shown in (5) also can be applied to light traveling along either of the other principal axes of the system, provided the polarization of the light beam is also parallel to a principal axis. These equations could easily be presented in a more compact form by expressing the orientation probabilities and experimental absorption cross-sections as vectors and the molecular cross-sections as a matrix. The orthogonal orientation probabilities are the projections of the orientation probability density ellipsoid onto the principal axes and integrated over all orientations.

$$
\begin{aligned}
& \sigma_{x}=\sigma_{\|} p_{x}+\sigma_{\perp} p_{y}+\sigma_{\perp} p_{z} \\
& \sigma_{y}=\sigma_{\perp} p_{x}+\sigma_{\|} p_{y}+\sigma_{\perp} p_{z}
\end{aligned}
$$




$$
\sigma_{z}=\sigma_{\perp} p_{x}+\sigma_{\perp} p_{y}+\sigma_{\|} p_{z}
$$

The sum of the orientation probabilities must be unity, as indicated in (6), and as probabilities, the values of each must be in the closed interval from 0 to 1 .

$$
p_{x}+p_{y}+p_{z}=1
$$

Therefore, in a steady state and in the absence of orienting stress, each probability must be one-third, so the cross-section for each direction reduces to the value expected for an isotropic sample, as is shown in (7).

$$
\sigma=\frac{\sigma_{\Perp}+2 \sigma_{\perp}}{3}
$$

Invoking these relationships results in the expressions for the LD and the dichroic increment ratio shown in (8) and (9). Complete alignment $\left(p_{y}=1\right)$ implies that the measured LD equals the molecular LD and that the dichroic increment ratio is -2 . However, a dichroic increment ratio of -2 does not imply complete alignment, because the equality of $p_{\mathrm{x}}$ and $p_{\mathrm{z}}$ always results in $R_{\mathrm{I}}=-2$. This well known result can be seen from (9) because if $p_{\mathrm{x}}$ and $p_{\mathrm{z}}$ are equal, the probability transferred to $p_{\mathrm{y}}$ will be exactly twice the probability lost by $p_{z}$. This is the distribution expected for stressors with axial symmetry, such as electric or magnetic fields. The discussion of the orientation of rigid rods in laminar gradient flow suggests that transfer of probability from $x$ to $y$ may be more efficient that from $z$ to $y$. In that case, $R_{\mathrm{I}}$ should be more negative than -2 and $p_{\mathrm{y}}>p_{\mathrm{z}}>p_{\mathrm{x}}$. The corresponding orientation probability density function will be a scalene ellipsoid. The ratio of the transferred probabilities is quantified in (10), which connects $p_{\mathrm{x}}$ and $p_{z}$ through the dichroic increment ratio, which can be determined experimentally.

$$
\begin{gathered}
\Delta \sigma_{\mathrm{LD}}=\sigma_{y}-\sigma_{z} \longrightarrow\left(\sigma_{\|}-\sigma_{\perp}\right)\left(p_{y}-p_{z}\right) \\
R_{\mathrm{I}}=\frac{\sigma_{\mathrm{y}}-\sigma}{\sigma_{z}-\sigma} \longrightarrow \frac{p_{y}-\frac{1}{3}}{p_{z}-\frac{1}{3}} \\
R_{\mathrm{I}}+1=-\frac{p_{x}-\frac{1}{3}}{p_{z}-\frac{1}{3}}
\end{gathered}
$$

3.4. The orientation probabilities and hydrodynamic parameters can be expressed as functions of the observable and molecular absorption parameters

The orientation probabilities are expressed in terms of the molecular $\left\{\sigma_{\|}, \sigma_{\perp}\right\}$ and experimental $\left\{\sigma_{y}, \sigma_{z}\right\}$ absorption cross-sections by solving the system of linear equations consisting of (5b), (5c) and (6), with the results shown in (11). 


$$
p_{\mathrm{x}}=\frac{\sigma_{\|}+\sigma_{\perp}-\left(\sigma_{\mathrm{y}}+\sigma_{\mathrm{z}}\right)}{\sigma_{\|}-\sigma_{\perp}}, \quad p_{\mathrm{y}}=\frac{\sigma_{\mathrm{y}}-\sigma_{\perp}}{\sigma_{\|}-\sigma_{\perp}}, \quad p_{\mathrm{z}}=\frac{\sigma_{\mathrm{z}}-\sigma_{\perp}}{\sigma_{\|}-\sigma_{\perp}}
$$

\subsection{Hydrodynamic parameters}

The optical parameters can be connected to the hydrodynamic properties of the system by introducing $\Theta$, the probability per unit time that a molecule or molecular fragment aligned with a particular axis will be realigned with one of the other axes due to random thermal motion, and $\beta_{x y}$ and $\beta_{z y}$, the probabilities per unit time that the aligning stress will shift molecules from being parallel to the $x$ or $z$ axes to become parallel to the $y$ axis. As defined here, $\Theta$ is analogous to the rotational diffusion constant and the values of $\beta_{x y}$ and $\beta_{z y}$ depend on the magnitude of the shear gradient, and must be zero in the steady state in the absence of shear. The thermal disorienting effect is presumed to result in equal probabilities per unit time of the transferred molecule going to each of the two other axes. The resulting expressions for the rates of change of each orientation probability are shown in (12), which also can be expressed in a more compact form in terms of vectors and a matrix.

$$
\begin{aligned}
& \frac{d p_{x}}{d t}=-\left(\Theta+\beta_{\mathrm{xy}}\right) p_{x}+\frac{\Theta}{2} p_{y}+\frac{\Theta}{2} p_{z} \\
& \frac{d p_{y}}{d t}=\left(\frac{\Theta}{2}+\beta_{\mathrm{xy}}\right) p_{x}-\Theta p_{y}+\left(\frac{\Theta}{2}+\beta_{\mathrm{zy}}\right) p_{z} \\
& \frac{d p_{z}}{d t}=\quad \frac{\Theta}{2} p_{x}+\frac{\Theta}{2} p_{y}-\left(\Theta+\beta_{\mathrm{zy}}\right) p_{z}
\end{aligned}
$$

In steady state flow, the derivatives must be zero. Dividing each term by $\Theta$ and defining orientation coefficients $c_{\mathrm{ij}}=\beta_{\mathrm{ij}} / \Theta$, which are dimensionless, results in a set of three equations that are not linearly independent because the sum of the decrease in $p_{x}$ and $p_{z}$ must equal the increase in $p_{y}$. However adding the requirement that the sum of the probabilities must be unity gives a set of three linearly independent equations that can be solved to obtain the expressions shown in (13).

$$
p_{x}=\frac{1}{3+2 c_{\mathrm{xy}}}, \quad p_{y}=1-p_{x}-p_{z}, \quad p_{z}=\frac{1}{3+2 c_{\mathrm{zy}}}
$$

Using these probabilities, the linear dichroism is shown in (14) and the dichroic increment ratio is shown in (15). As above, $R_{\mathrm{I}}$ does not depend on optical parameters, even though it is determined by absorption measurements.

$$
\mathrm{LD} \equiv \sigma_{y}-\sigma_{z}=\left(\sigma_{\|}-\sigma_{\perp}\right)\left(p_{y}-p_{z}\right) \longrightarrow\left(\sigma_{\|}-\sigma_{\perp}\right)\left(1-\frac{1}{3+2 c_{\mathrm{xy}}}-\frac{2}{3+2 c_{\mathrm{zy}}}\right)
$$




$$
R_{\mathrm{I}}=\frac{-\left(2-\frac{3}{3+2 c_{\mathrm{xy}}}-\frac{3}{3+2 c_{\mathrm{zy}}}\right)}{\left(1-\frac{3}{3+2 c_{\mathrm{zy}}}\right)}
$$

Rearranging (15) demonstrates that $c_{\mathrm{zy}}$ can be expressed in terms of $c_{\mathrm{xy}}$ and the experimentally determined value of $R_{\mathrm{I}}$, as shown in (16).

$$
c_{\mathrm{zy}}=\frac{-3 c_{\mathrm{xy}}}{3\left(1+R_{\mathrm{I}}\right)+2\left(2+R_{\mathrm{I}}\right) c_{\mathrm{xy}}}
$$

\section{Analysis and discussion}

The equivalent orthogonal orientation model correctly represents properties of partially oriented samples for a light beam propagating parallel to one and with polarization parallel to either of the other principal axes defined above. It is not realistic in the sense that molecules do not orient exactly parallel to one of the principal axes of the coordinate system. But the concept of orthogonal projection probabilities made it possible to write equations (5) and (6) by inspection, the compact notation facilitated the subsequent derivations, and the model provides results consistent with well established properties of isotropic samples, e.g. (7). Equations (5) are equivalent to the well established tensor-based theories provided the measurement uses the standard geometry for an LD experiment; these correlations are shown in Table 1. In (5) the orthogonal projection probabilities were defined functionally, but they can be interpreted as the projections onto each principal axis of the cosine-square weighted probability density for the alignment of each orientable unit averaged over all angles, which is implicit in the notation of Hofrichter and Eaton [19].

Table 1. Correspondence between the orthogonal projection probabilities as defined in this report with the corresponding terms in tensor based models.

\begin{tabular}{ccccc}
\hline $\begin{array}{c}\text { equivalent } \\
\text { orthogonal } \\
\text { orientation } \\
\text { model }\end{array}$ & $\begin{array}{c}\text { Peterlin } \\
{[21]}\end{array}$ & $\begin{array}{c}\text { Wada } \\
{[18]}\end{array}$ & $\begin{array}{c}\text { Hofrichter } \\
\text { and Eaton } \\
{[19]}\end{array}$ & $\begin{array}{c}\text { Nordén, } \\
\text { Rodger and } \\
\text { Dafforn } \\
{[1]}\end{array}$ \\
\hline$p_{\mathrm{x}}$ & $\frac{1}{3}+\frac{8 \pi}{5} a_{11}-\frac{2 \pi}{15} a_{10}$ & $\frac{\mathrm{K}_{1}+\mathrm{K}_{2}}{2}$ & $\left\langle\operatorname{Cos}^{2} \xi\right\rangle$ & $\frac{2 S_{x x}+1}{3}$ \\
$p_{\mathrm{y}}$ & $\frac{1}{3}-\frac{8 \pi}{5} a_{11}-\frac{2 \pi}{15} a_{10}$ & $\frac{\mathrm{K}_{1}-\mathrm{K}_{2}}{2}$ & $\left\langle\operatorname{Cos}^{2} \psi\right\rangle$ & $\frac{2 S_{y y}+1}{3}$ \\
$p_{\mathrm{z}}$ & $\frac{1}{3}+\frac{4 \pi}{15} a_{10}$ & $1-\mathrm{K}_{1}$ & $\left\langle\operatorname{Cos}^{2} \zeta\right\rangle$ & $\frac{2 S_{z z}+1}{3}$ \\
\hline
\end{tabular}




\subsection{Properties and applications of the dichroic increment ratio}

The dichroic increment ratio provides information on the distribution of orientable molecules not obtainable from unpolarized absorption, the simple linear dichroism difference, or the reduced LD. The linear dichroism is dependent on both the degree of orientation and the intrinsic average dichroism of the macromolecules, as demonstrated by (8) and (14). However, the dichroic increment ratio depends only on the orientation parameters, and is independent of the molecular absorption cross-sections, as shown in (9) and (15), which is consistent with the experimental results of Gray and Rubenstein [3]. This is an interesting result considering that the ratio is obtained from measurements of absorption. The same ratio should be found for different transitions measured at different wavelengths, a result which is also consistent with the results of Gray and Rubenstein and experiments performed with rectangular flow cells, as shown below.

Absorption data from early publications reflect the scalene distribution of DNA and DNA complexed with several drugs created by hydrodynamic shear in rectangular cross-section flow cells, as summerized in Table 2 and demonstrated graphically in Figure 3. (The optical pathlength of rectangular flow cells was typically a few tens of microns, with a width of about one and height of a few centimeters. The top and bottom were tapered to permit connections to flexible tubing.) The dichroic increment ratio was not computed in these publications; the avaibility of these data are a fortitious artifact of the way that LD was measured in a spectrophotometer. The light emerging from a monochromator is partially linearly polarized due to nonnormal reflections from the various optical elements. Therefore, the intensity of the incident light is different for different planes of polarization of the light beam. To correct for the influence of this partial polarization, the flow-on, flow-off absorbance was measured for each orientation of the polarizer. The absorption difference for light polarized perpendicular to the orientation direction was then subtracted from that of the parallel orientation to give the LD. Data were presented as "reduced" dichroisms by dividing by the unpolarized absorbance, thus eliminating the dependence of the result on concentration and pathlength (small unbound and therefore unorientable ligands contribute to the total absorption but not to the LD or the dichroic increment ratio). Later publications usually presented only the polarized absorption differences, and omitted the data from intermediate steps; Gray and Rubenstein[20] are an important exception, as they explicitly calculated the dichroic increment ratio. The flow-cell data cited in Table 2 indicate that the magnitude of the dichroic increment ratio is consistently greater than 2 , i.e., the LD ratio is numerically less than -2 . Because the expression on the right-hand side of (15) does not contain any wavelength-dependent terms, it should be the same for all wavelengths to within experimental uncertainty, and the data in Table 2 are consistent with this prediction, assuming an uncertainty in the reduced flow-on, flow-off LD large enough to change the value of the least significant digit to the next larger or smaller numerical value. The data for distamycin A are particularly relevant because the sign of the LD changes for the wavelengths dominated by the DNA compared to that of the distamycin A, but the dichroic increment is essentially the same. 
The precision of the data summarized in Table 2 was adequate for the purposes of the publications in which they appeared. Significantly improved precision can be expected from experiments in which the goal is to obtain accurate values of the dichroic increment ratio. Cavalieri et al. [2], who appear to have assumed that the distribution of DNA induced by flow shear is inherently uniaxial, stated that the dichroic increment ratio for DNA should be -2 and that this result had been "... verified experimentally ...", but did not present numerical data. Unlike a Couette cell, the experiments of Cavalieri et al. and those cited in Table 2 involved DNA flowing through a cell with a rectangular cross-section, so the flow gradient was not uniform. According to the Navier-Stokes equation, which applies to the flow of an incompressible fluid between two stationary flat plates of infinite extent, the distribution of flow velocities is parabolic. The velocity distribution in a rectangular flow cell with an optical path length of tens of microns and a width of one centimeter is therefore expected to be approximately parabolic, with the maximum velocity in the center of the cell and decreasing to zero at the inside front and back surfaces. Therefore, the gradient is zero in the center of the cell and increases linearly in magnitude in each direction along the light path. Therefore the resulting dichroism is an average over gradients from zero up to the maximum. The average shear, and hence the average degree of orientation that can be achieved without inducing turbulence, will therefore be lower than can be achievable with a Couette cell, in which the gradient is ideally constant along the optical path. The fact that Gray and Rubenstein, using a Couette cell, found the dichroic increment ratio to be 2 to within experimental error, means that the alignment they achieved was effectively uniaxial.

Table 2. Flow linear dichroism, dichroic increment ratio, and isotropic absorption data for DNA and three complexes of DNA with drugs from the indicated publications. The DNA was from chicken erythrocytes (CE), calf thymus (CT), bacteriophage T2 and T5, or an unstated source (U). DNA was oriented in either a flow cell with a rectangular cross-section, $\mathrm{R}$ or a Couette cell, $\mathrm{C}$. 


\begin{tabular}{|c|c|c|c|c|c|c|c|c|c|}
\hline DNA & Ligand & $\begin{array}{c}\lambda \\
(\mathrm{nm})\end{array}$ & $\frac{A_{y}-A}{A}$ & $\frac{A_{z}-A}{A}$ & $\frac{A_{y}-A_{z}}{A}$ & $\frac{A_{y}-A}{A_{z}-A}$ & $A$ & Cell & Ref \\
\hline $\mathrm{CE}$ & none & 260 & -0.19 & 0.086 & -0.28 & -2.21 & & $\mathrm{R}$ & [6] \\
\hline $\mathrm{CE}$ & quinacrine & 260 & -0.24 & 0.11 & -0.35 & -2.18 & 1.422 & $\mathrm{R}$ & {$[6]$} \\
\hline $\mathrm{CE}$ & quinacrine & 432.5 & -0.25 & 0.12 & -0.37 & -2.08 & 0.240 & $\mathrm{R}$ & {$[6]$} \\
\hline $\mathrm{CE}$ & quinacrine & 455 & -0.24 & 0.11 & -0.35 & -2.18 & 0.213 & $\mathrm{R}$ & {$[6]$} \\
\hline $\mathrm{U}$ & $\begin{array}{l}\text { chloroquin } \\
\mathrm{e}\end{array}$ & 260 & -0.21 & 0.09 & -0.30 & -2.33 & & $\mathrm{R}$ & [7] \\
\hline $\mathrm{U}$ & $\begin{array}{l}\text { chloroquin } \\
\mathrm{e}\end{array}$ & 349 & -0.21 & 0.08 & -0.29 & -2.63 & & $\mathrm{R}$ & [7] \\
\hline $\mathrm{CT}$ & $\begin{array}{l}\text { distamycin } \\
\text { A }\end{array}$ & 259 & -0.22 & 0.09 & -0.31 & -2.44 & & $\mathrm{R}$ & [9] \\
\hline CT & $\begin{array}{l}\text { distamycin } \\
\text { A }\end{array}$ & 320 & 0.19 & -0.08 & 0.27 & -2.38 & & $\mathrm{R}$ & [9] \\
\hline $\mathrm{T} 2$ & none & 215 & \multirow{2}{*}{\multicolumn{3}{|c|}{ Varies by wavelength }} & -2.0 & & & \\
\hline T5 & none & $\begin{array}{c}\text { to } \\
290\end{array}$ & & & & $\begin{array}{c} \pm \\
0.1\end{array}$ & & C & [3] \\
\hline
\end{tabular}

\subsection{An example that requires more than the orthogonal projection model}

The orthogonal projection model does not predict correct results when the polarization or the propagation of light are not parallel to a primary axes. As mentioned above, the alignment probabilities only correlate with the diagonal elements of the orientation matrix; the off-diagonal elements are not considered. This is apparent in the data in Figure 3. Using the orthogonal projection model, the expression for the absorption of light traveling in the positive $\mathrm{x}$ direction and polarized at an angle $\chi$ with respect to the orientation axis, the flowinduced LD at $257 \mathrm{~nm}$ would be as shown by the dashed line. But this expression results in a poor fit to the experimental data for angles not near $0^{\circ}$ or $90^{\circ}$. The solid curve in Figure 3 was obtained by adding an additional term proportional $\operatorname{Sin}^{2} \chi \operatorname{Cos}^{2} \chi$ to this expression. 


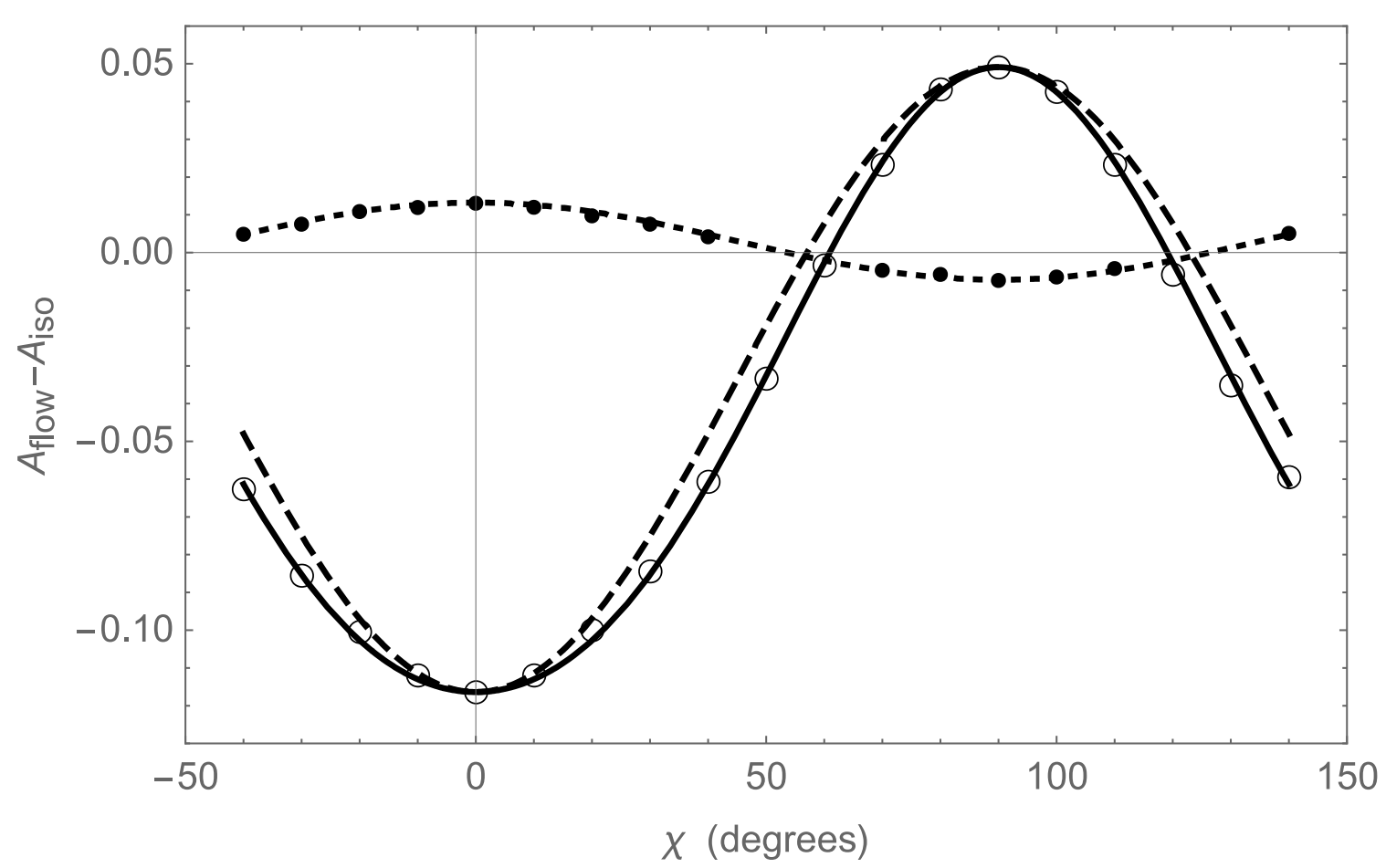

Figure 3. Dichroism of DNA complexed with Distamycin A as a function of the angle of polarization of the light beam, $\chi$ relative to the direction of flow for wavelengths of 259 (O) and $320(\bullet) \mathrm{nm}[9]$. Aflow is absorption measured with the sample flowing and the corresponding value of $A_{\text {iso }}$ was measured with the sample at rest and with the same setting of the polarizer. The dashed line is a plot of $\left(A_{0}{ }^{\circ}-A\right) \operatorname{Cos}^{2}[\chi]+\left(A_{90^{\circ}}-A\right) \operatorname{Sin}^{2}[\chi]$, where the values of the coefficients are from Table 2 . The poor fit for angles not near $0^{\circ}$ or $90^{\circ}$ is discussed in the text. The solid line is the same expression to which was added -0.055 $\operatorname{Cos}^{2}[\chi] \operatorname{Sin}^{2}[\chi]$, significantly improving the fit to the experimental data.

\section{Protocols for measuring flow-oriented linear dichroism}

Several protocols have been reported in measurements of shear flow-induced LD of DNA. The early experiments of Lerman [6] reported the difference in absorption between the flowing sample and the same sample in the absence of flow. This parameter was reported for light polarized both parallel and perpendicular to the direction of flow and propagating in the direction parallel to the shear gradient. The sample was pumped through a rectangular cell with an optical path length of $27 \mu \mathrm{m}$, so the shear gradient was not uniform. Krey and Hahn [9] used a similar configuration, but measured the absorption for a range of polarization angles, as shown in Figure 3. For studies investigating the binding of a ligand to DNA, the LD is measured at different wavelengths to separate absorption of the DNA and the ligand, but only a few wavelengths were used presumably because the experiments were labor-intensive. Subsequently, only the difference between the absorption of the flowing sample for light polarized parallel and perpendicular to the direction of flow was reported. Wada [22] introduced the use of Couette cells to induce flow with a linear shear gradient to studies of DNA. He also proposed a spectrometer that would measure absorption for these two polarizations automatically, but the ability 
to easily measure the LD across a broad spectrum was not readily feasible until it was realized that with minor adjustments, a dichrometer designed for the measurement of CD can also measure LD. The analysis presented above demonstrates that useful information is lost when the flow-on, flow-off difference is not recorded for both the vertical and horizontal polarizations. Thus, there are limits on LD measurement made with a dichrometer if such information is desired.

\subsection{Extracting scalene distribution parameters with a dichrometer}

The data needed to compute the dichroic increment ratio can be obtained with a spectrophotometer equipped with a rotatable polarizer and either a flow cell or a Couette cell as described by the papers that provided the data shown in Table 3 . But a modern dichrometer operating in LD mode is capable of far higher sensitivity for the measurement of the LD difference signal than can be achieved with a spectrophotometer. Dichrometers also can acquire the LD across a broad spectrum of wavelength quickly. Moving the Couette cell to a different instrument would be inconvenient, probably require separate mounting fixtures, and make it difficult to be sure the two measurements are performed under exactly the same conditions. Thus using a dichrometer to measure both the LD and the dichroic increment ratio is desirable. Whether this is practical depends on the details of the instrument.

The simplest situation would be a bespoken dichrometer fitted with a separate polarizer. While in principal the polarizer can be set at any angle so long as the polarization of the beam is oriented at an angle of $45^{\circ}$ with respect to the principal axis of the optical element of the photoelastic modulator (PEM), in practice the polarizer is usually set to transmit light polarized parallel to either the vertical or horizontal axis of the instrument. Fortunately, that is exactly the condition required for application of the equivalent orthogonal orientation model. It would not be difficult to arrange for the polarizer to be rotatable and to turn off the PEM so the light reaching the sample could be made to have either polarization. In bespoken instruments, it also should be possible to deactivate the high-voltage servo system that adjusts the high-voltage applied to the photomultiplier and to set this voltage manually. Then all that is required to measure the numerator and denominator of the dichroic increment ratio is to record the photomultiplier signal for both polarizations with the Couette cell operating and when it is turned off and computing the corresponding absorbances as indicated in (2). Because only the absorbance difference is needed, it is not necessary to measure $I_{0}$. The original emission and polarization spectrometer [13] was equipped with both a PEM and a mechanical modulator (chopper), which combined with a lock-in amplifier could measure light intensities with high precision. The determination of the dichroic increment ratio should have to be performed at only one wavelength, so the computation is not onerous. Presumably, most of the synchrotron radiation CD/LD beamlines should be capable of performing the measurements indicated above, although those that normally depend of the intrinsic polarization of the photon beam generated in a bending magnet may have to add a separate polarizer and increase the aperture to accept more out-of-plane radiation. 
The situation with commercial dichrometers is likely to be more difficult for two reasons. The high-voltage servo system works to maintain constant time-average current from the photomultiplier. It thus serves as a safety device to protect photomultipliers from high currents and Ohmic heating resulting from exposure to intense light. If the photocathode is exposed to, for example, room light, the servo circuit rapidly decreases the high voltage. Thus manufactures may be reluctant to make it possible for end users to control the high voltage manually. However, absorbance differences can be determined by observing the change in the high voltage between the two states that are being studied while the servo-circuit is operating. This follows because the logarithm of the gain of a photomultiplier is approximately proportional to the applied high voltage, an effect that makes possible the measurement of the absorption spectrum of the sample at the same time the CD or LD are recorded [15]; also see [23], [24], or [17]. For dichrometers that use a separate polarizer, all that would be needed is the ability to rotate it through $90^{\circ}$.

Some of the most popular commercial dichrometers, however, use double monochromators with crystalline prisms to both disperse the spectrum and select the polarization. In such instruments, rotating the plane of polarization of the light beam would be more complicated than rotating a polarizer. It may still be possible to obtain the data for the determination of the LD ratio with such instruments, provided the PEM can be turned on and off and its phase amplitude adjusted. As shown in Table 3, for a dichrometer that generates a horizontally polarized incident beam, the numerator of the dichroic increment ratio is the difference in absorption between the Couette cell on and off when the PEM is off, and this is just the differences in the values of the pseudo absorptions (denoted by ${ }^{p} A$, i.e., the high voltage values recorded with the servo control operating), as shown in (17). With the PEM operating, and assuming the phase is set to eliminate the $J_{0}$ term [24], the difference in the pseudo absorptions gives the average of the $A_{\mathrm{y}}$ and $A_{\mathrm{z}}$, which, when combined with the result for the PEM-off values, is enough to determine the dichroic increment ratio, as shown in (18).

$$
\begin{gathered}
\Delta A_{-} \equiv{ }^{p} A_{-+}-{ }^{p} A_{--}=A_{y}-A \\
\Delta A_{+} \equiv{ }^{p} A_{++}-{ }^{p} A_{+-}=\frac{A_{y}+A_{z}}{2}-A \\
R_{\mathrm{I}} \equiv \frac{A_{y}-A}{A_{z}-A}=\frac{\Delta A_{-}}{2 \Delta A_{+}-\Delta A_{-}}
\end{gathered}
$$

Table 3. Absorption coefficients for four states of a dichrometer equipped with a PEM and a Couette cell where the plane of polarization on the light incident on the PEM and the direction of preferred orientation are both along the $y$ axis. Other expressions apply to different combinations of orientations. $A_{\mathrm{i}}$ is the absorption coefficient for light polarized 
parallel to the $\mathrm{i}^{\text {th }}$ axis and $A$ is the isotropic absorbance.

\begin{tabular}{cccc}
\hline & \multicolumn{3}{c}{ Couette Cell } \\
& & On & Off \\
PEM & On & $A_{++}=\frac{A_{y}+A_{z}}{2}$ & $A_{+-}=A$ \\
& Off & $A_{-+}=A_{\mathrm{y}}$ & $A_{--}=A$ \\
\hline
\end{tabular}

\section{Conclusions}

The dichroic increment ratio can be used to determine if the distribution of DNA in a particular experiment is effectively uniaxial, and if it is not, to quantify the degree of to which the distribution is scalene. This ratio is expected to be -2 for axial orientations, but may be more negative for hydrodynamic orientation because molecules or molecular segments oriented perpendicular to both the direction of flow and the flow gradient are oriented less efficiently. Although determined by polarized absorption spectroscopy, the dichroic increment ratio is independent of the wavelength of the light and the optical properties of the alienable system, but is a function of the magnitude of the shear gradient. Measurement of the dichroic increment ratio as a function of the rotation rate, and hence the magnitude of the shear, may provide information on the changes in conformation of DNA as it is aligned. In addition to facile measure of linear dichroism, it may be possible to measure the dichroic increment ratio in a modern dichrometer equipped with a photoelastic modulator, depending on the extent that the user can control the high voltage applied to the photomultiplier and the operation of the modulator.

The equivalent orthogonal orientation model permits characterization of the distribution of molecules oriented by hydrodynamic flow or other means that correctly replicates known optical properties of such systems. This model can be applied to the distribution of orientations of rigid linear molecules or other structures and also flexible structures, which are distorted by shear stress. The distribution of partially aligned molecules can be described by the dimensionless ratio of two parameters that reflect the competing effects of shear-induced orientation and rotational diffusion. The relative values of these parameters can be determined experimentally by measuring the ratio of the flow-induced difference in absorption for light polarized parallel and perpendicular to the direction of orientation, i.e. the dichroic increment ratio.

\section{Acknowledgments}

I thank Anna Krey for sharing with me experimental results prior to their publication. Supported by Augusta University, a unit of the University System of 
Georgia. Brookhaven National Laboratory is supported by the U. S. Department of Energy.

\section{References}

[1] B. Nordén, A. Rodger, T.R. Dafforn, Linear dichroism and circular dichroism: a textbook on polarized spectroscopy, Royal Society of Chemistry, Cambridge, 2010.

[2] L.F. Cavalieri, B.H. Rosenberg, M. Rosoff, Flow Dichroism and its Application to the Study of Deoxyribonucleic Acid Structure, J. Am. Chem. Soc., 78 (1956) 52355238.

[3] D.M. Gray, I. Rubenstein, Ultraviolet dichroic ratio of DNA from T2 and T5 bacteriophages, Biopolymers, 6 (1968) 1605-1631.

[4] A. Wada, Chain regularity and flow dichroism of deoxyribonucleic acids in solution, Biopolymers, 2 (1964) 361-380.

[5] R. Marrington, T.R. Dafforn, D.J. Halsall, A. Rodger, Micro-volume couette flow sample orientation for absorbance and fluorescence linear dichroism, Biophys. J., 87 (2004) 2002-2012.

[6] L.S. Lerman, The sructure of the DNA-acridine complex, Proc. Natl. Acad. Sci. U. S. A., 49 (1963) 94-102.

[7] R.L. O'Brien, J.L. Allison, F.E. Hahn, Evidence for intercalation of chloroquine into DNA, Biochim. Biophys. Acta, 129 (1966) 622-624.

[8] M. Kodama, The Interaction of Mitomycin C with Deoxyribonucleic Acid in vitro, The Journal of Biochemistry, 61 (1967) 162-167.

[9] A.K. Krey, F.E. Hahn, Studies on the complex of distamycin a with calf thymus DNA FEBS Letters, 10 (1970) 175-178.

[10] F. Dall'Acqua, M. Terbojevich, S. Marciani, D. Vedaldi, M. Recher, Investigation on the dark interaction between furocoumarins and DNA, Chemico-Biological Interactions, 21 (1978) 103-115.

[11] M. Shahbaz, N.E. Geacintov, R.G. Harvey, Noncovalent intercalative complex formation and kinetic flow linear dichroism of racemic syn- and antibenzo[a]pyrenediol epoxide-DNA solutions, Biochem., 25 (1986) 3290-3296.

[12] C. Nagata, M. Kodoma, Y. Tagashira, A. Imamura, Interaction of polynuclear aromatic hydrocarbons, 4-nitroquinoline 1-oxides, and various dyes with DNA, Biopolymers, 1966 (1966) 409-427.

[13] J.C. Sutherland, G.D. Cimino, J.T. Lowe, An Emission and Polarization Spectrometer for Biophysical Spectroscopy, Rev. Sci. Instrum., 47 (1976) 358-360.

[14] D. Canet, K. Doering, C.M. Dobson, Y. Dupont, High Sensitivity Fluorescence Anisotropy Detection of Protein Folding Events: Application to alpha-Lactalbumin, Biophys. J., 80 (2001) 1996-2003. 
[15] J.C. Sutherland, P.C. Keck, K.P. Griffin, P.Z. Takacs, Simultaneous measurement of absorption and circular dichroism in a synchrotron spectrometer, Nuc. Instr. Meth., 195 (1982) 375-379.

[16] J.C. Sutherland, Simultaneous measurement of circular dichroism and fluorescence polarization anisotropy, in: G.E. Cohn (Ed.) Clinical Diagnostic Systems: Technologies and Instrumentation, SPIE, 2002, pp. 126-136.

[17] J.C. Sutherland, Measurement of the Circular Dichroism of Electronic Transitions, in: N. Berova, P. Polavarapu, K. Nakanishi, R.W. Woody (Eds.) Comprehensive Chiroptical Spectroscopy, John Wiley \& Sons, New York, 2012, pp. 37-63.

[18] A. Wada, Dichroic spectra of biopolymers oriented by flow, Applied Spectroscopy Reviews, 6 (1972) 1-30.

[19] W.A. Eaton, J. Hofrichter, Linear Dichroism of Biological Chromophores, Ann. Rev. Biophys. Bioeng., 5 (1976) 511-560.

[20] D.M. Gray, The Ultraviolet Dichroic Ratio of DNA from T5 and T2 Bacteriophages Yale University, 1968, pp. 212.

[21] A. Peterlin, Optical effects in flow, Annual Review of Fluid Mechanics, 8 (1976) 35-55.

[22] A. Wada, S. Kozawa, Instrument for the studies of differential flow dichroism of polymer solutions, Journal of Polymer Science Part A, 2 (1964) 853-864.

[23] J.C. Sutherland, Circular dichroism using synchrotron radiation: from ultraviolet to x-rays, in: G.D. Fasman (Ed.) Circular Dichroism and the Conformational Analysis of Biomolecules, Plenum Press, New York, 1996, pp. 599633.

[24] J.C. Sutherland, Measurement of Circular Dichroism and Related Spectroscopies with Conventional and Synchrotron Light Sources: Theory and Instrumentation, in: B.A. Wallace, R.W. Janes (Eds.) Modern Techniques for Circular Dichroism and Synchrotron Radiation Circular Dichroism Spectroscopy, IOS Press, Amsterdam, 2009, pp. 19-72. 